\title{
Movilidad en bus como sistema de transporte urbano
}

\section{Mobility in bus as urban transport system}

\author{
Javier Celestino Villegas Mayorga ${ }^{1}$
}

\section{Resumen}

El presente artículo tiene como objetivo el análisis de la movilidad en bus como sistema de transporte urbano en Bogotá D.C, frente a las diferentes ciudades capitales europeas (Berlín, Madrid, París, Roma y Londres), puesto que el transporte público juega un papel clave en cómo se forman las sociedades urbanas modernas, en particular la ciudad de Bogotá, demostrando que la mejora urbana se da por reacción del mercado, por lo que esos casos son contrastados con las experiencias de las ciudades citadas, en cuanto a la planeación urbana integrada de formas compactas, uso mixto de suelo y construcción de espacios amigables para las caminatas y la convivencia social, en correlación con un transporte público integrado, de alta calidad y capacidad, que ayuda a concretarla. Al analizar la movilidad de las personas en las ciudades europeas se enfatiza que ésta se realiza en diferentes circunstancias de comodidad, seguridad y tiempo, por lo que el uso excesivo del transporte privado hace necesario el fomento del transporte público urbano, además los retos actuales de organización del transporte urbano pasan por determinar el lugar que debe ocupar el automóvil y la accesibilidad tarifaria, temporal y territorial del transporte público colectivo, pues el sistema

Asesor y evaluador de proyectos en Tesis Colombia

\footnotetext{
${ }^{1}$ Politólogo. Universidad de los Andes. Venezuela villegasjavier92@gmail.com
} AGLALA ISNN 2215-7360

2015; 6 (1): 102-120 
CÓDIGO JEL: R40

Fecha de recepción: Mayo de 2015 / Fecha de aceptación en forma revisada: Agosto 2015

de transporte tiene una gran afinidad y puede llegar a conseguir que un área como Bogotá tenga identidad territorial propia.

Palabras Clave: Movilidad; Sistema de Transporte Urbano; Identidad Territorial; Crecimiento Urbanístico.

\begin{abstract}
This paper aims to analyze mobility in bus and urban transport system in Bogota DC, against the different capitals Europe (Berlin, Madrid, Paris, Rome and London), since public transport plays a key role how modern urban societies are formed, particularly Bogota, showing that urban improvement occurs by reaction of the market, so that such cases are contrasted with the experiences of those cities, in terms of urban planning integrated compact forms, mixed land use and construction of friendly spaces for walks and social life, in correlation with an integrated public transport, high quality and capacity, which helps achieve it. When analyzing the mobility of people in European cities it is emphasized that this is done in different circumstances of comfort, safety and time, so that excessive use of private transport necessitates the promotion of urban public transport, as well the current challenges Urban organization go through determine the rightful place of the car and tariff accessibility, temporal and territorial of public transport because the transport system has a high affinity and can even get you an area like Bogotá have own territorial identity.
\end{abstract}

Keywords: Mobility; urban transport system; territorial identity; urban growth.

AGLALA ISNN 2215-7360

2015; 6 (1): 102-120 


\section{Introducción}

La movilidad urbana se perfila en la actualidad como un desafío para las personas que hacen vida dentro de las ciudades, y que a su vez, realizan diferentes actividades que son de su interés. En este caso, el traslado de las personas dentro de la ciudad pueden realizarlo caminando o utilizando vehículos de transporte, puesto que los servicios de transporte han sido considerados como servicios públicos, garantizando la accesibilidad a la movilidad urbana de los todos los ciudadanos (Miralles-Guasch y Cebollada, 2012).

Todo ello, evidencia que la movilidad urbana aumenta a través del acelerado crecimiento de la urbanización de las sociedades contemporáneas, lo cual deja en evidencia la necesidad de mantener y cuidar los espacios para una mejor calidad de vida, incluyendo a su vez, las condiciones adecuadas de movilidad de las personas. Por tal razón, en las grandes metrópolis como Bogotá se registra algunos problemas sociales, económicos y ambientales relacionados con el desplazamiento de sus habitantes, los cuales se consideran como las necesidades que posee el individuo y que han sido limitados de los servicios de infraestructura adecuada (Rogat, 2009).

Concretamente, la infraestructura y servicios de transporte juegan un papel importante como herramienta que logra revertir el problema del acceso a los servicios de infraestructura de transporte, puesto que esta desigualdad impacta en el dinamismo económico de la ciudad y en la calidad de vida de sus habitantes. Por ello, el Estado es garante de resolver los problemas que presentan las comunidades, desarrollando nuevos esquemas de cobertura y calidad de la infraestructura para de esta forma incrementar la calidad de vida de sus habitantes (Ribeiro, 2000).

AGLALA ISNN 2215-7360

2015; 6 (1): 102-120 
Bajo esta premisa, se tiene que el objetivo fundamental del presente estudio es analizar la movilidad en bus como sistema de transporte urbano en Bogotá, es decir partiendo del concepto movilidad urbana se logra comprender con otros modelos de sistema de transporte urbano. En base a lo expuesto, la investigación resulta relevante puesto que a través del presente estudio se aspira brindar información necesaria sobre la problemática del acceso a los servicios de infraestructura de transporte; así como alternativas, propuestas y recomendaciones concretas en la implementación de un sistema de transporte público en Bogotá.

Como es sabido, la ciudad de Bogotá, y en general Colombia, se encuentran en un proceso de cambio, crecimiento y transformación, debido al crecimiento económico que el país viene experimentado, de acuerdo con la visión del futuro para Bogotá, convirtiéndola en el centro urbanístico de atracción en América Latina(Perdomo, Mendoza, Mendieta y Baquero, 2007).

De igual forma, Bogotá ha ido creciendo en cuanto a infraestructura vial se refiere, dotadas de grandes autopistas y carreteras que comunican las diferentes partes de la ciudad, la cual la hace competitiva con el resto de las grandes ciudades de los países desarrollados como Berlín, Madrid, París, Roma y Londres, por sus características que se podrían esperar de una ciudad moderna (Hidalgo y Yepes, 2004).

Se tiene en cuenta que el desafío de las grandes ciudades como Bogotá es el transporte público, lo cual representa la única opción viable de movilización en los sectores más desposeídos de la sociedad (Borja, 2003). Por lo que es transcendental modificar el sistema vial central de la ciudad de Bogotá, para conseguir una movilidad urbana más equitativa, eficiente y ambientalmente sostenible, que pueda elevar la calidad de vida en la población, el nivel de accesibilidad y de movilidad de sus ciudadanos.

AGLALA ISNN 2215-7360

2015; 6 (1): 102-120 
Bajo esta apreciación, se afirma que el sistema urbano de movilidad es un instrumento de integración espacial que mejora la calidad de vida urbana, las condiciones de los desplazamientos que tienen las personas y los impactos ambientales que el tráfico ocasiona en el medio natural y urbano. Brindando de esta forma, oportunidades en cuanto a la movilidad cotidiana de la población (Jiménez 2012).

Por ello, movilizarse por la ciudad permite integrarse en la vida urbana y de moverse dentro de un espacio público, acordando un conjunto de medidas que garanticen a todos los usos del espacio urbano y la fluidez del transporte público. Desde el punto de vista organizativo, el sistema de transporte público toma en consideración la planeación motivado al papel que representa la estructuración del espacio urbano, debido a la influencia directa de las condiciones generales del proceso de urbanización de la ciudad (Rodríguez y Targa, 2004).

Por tal motivo, el problema se centra en investigar sobre la movilidad de bus como sistema de transporte público para el ámbito metropolitano de Bogotá zona centro frente a las diferentes ciudades capitales europeas (Berlín, Madrid, París, Roma y Londres).En este sentido, es importante conocer el marco organizativo del sistema de transporte público, definiendo los servicios y planificación de las infraestructuras viales.

Sin embargo, la infraestructura vial de la ciudad de Bogotá ha generado el aumento de la demanda en el transporte público y privado en las calles, debido a un alto movimiento urbano, trayendo como consecuencia que el tiempo de viaje se vea prolongado debido a la sobre carga de tráfico en las principales vías de la ciudad. Otro problema que afecta de manera negativa la infraestructura vial de Bogotá es el estado de las vías, puesto que las estadísticas del Instituto de 
Desarrollo Urbano (IDU) confirman que las condiciones de la infraestructura vial de la ciudad esté en condiciones inestables (Banco Mundial, 2009).

De lo anteriormente expuesto, se puede inferir que la ciudad de Bogotá en la actualidad enfrenta la necesidad de manejar las redes de infraestructura vial como parámetro de efectividad para determinar el grado de uso, capacidad disponible y relación volumen de congestión vehicular. Por esta razón, el desafío consiste en llevar a cabo un desarrollo de programas de monitoreo, reportaje y verificación para identificar las fallas de la infraestructura vial, de manera que se pueda acondicionar al actual volumen de tráfico y al del futuro (Ávila, 2003).

De igual forma, es importante mejorar la experiencia del usuario de transporte público, basándose en la satisfacción de los usuarios y promoviendo un mayor uso de los servicios de transporte. Puesto que, es importante mejorar el transporte público a través del coste, infraestructura, seguridad de las personas y el acceso que éstas tengan al servicio, convirtiéndolo en una alternativa más interesante que el automóvil (Acevedo, Bocarejo, Echeverry, Lleras, Ospina y Rodríguez, 2009).

Es por esta razón, que este estudio tiene como propósito principal ayudar a mejorar la movilidad en bus como sistema de transporte urbano en Bogotá, tomando en consideración las experiencias de modelos internacionales, estableciendo de esta forma los criterios para la movilidad del sistema de transporte público, para mejorar las condiciones de transporte en la zona de estudio.

Al extender estas afirmaciones a una investigación realizada, Perdomo et. al (2010), referente a movilidad en bus como sistema de transporte en Bogotá, surge la siguiente 
interrogante: ¿Cuál ha sido la experiencia de Bogotá, en cuanto a la movilidad en bus como sistema de transporte urbano?

Para la realización de este estudio se consultaron varias obras que guardan relación con el objeto de esta investigación, entre las cuales se pueden mencionar las siguientes:

Figueroa y Castañeda (2013), realizaron una investigación titulada "Caracterización de los criterios logísticos clave de transporte urbano de pasajeros para el Barrio Belisario Caicedo de la Comuna 20 en Santiago de Cali", cuyo objetivo fue Establecer los criterios logísticos claves para evaluar la eficiencia del transporte urbano de pasajeros, adecuado a zonas de múltiples servicios públicos en Colombia, específicamente en el barrio Belisario Caicedo de la Comuna 20 de la ciudad de Santiago de Cali. La metodología empleada se basó en un análisis de campo de una zona de estudio específica y, en donde se levantaron datos por medio de una encuesta piloto sobre temas de movilidad, infraestructura y nivel se servicio de los diferentes modos que convergen en este punto específico.

Los resultados del estudio evidencian que: (i) los problemas de movilidad no sólo se presentan en los países en vía de desarrollo sino que también en los grandes centros urbanos del mundo; (ii) se generó una propuesta taxonómica de los criterios logístico de movilidad para los sistemas de transporte urbano de pasajeros y; (iii) se presentó el estado actual de la movilidad en un sector específico de la ciudad de Santiago de Cali.

Seguidamente, Martínez (2012) efectuó un estudio titulado "Estrategias para promover la accesibilidad, cobertura y calidad en el sistema de transporte público urbano para la población con discapacidad física: Caso Bogotá”, teniendo como objetivo principal presentar una estrategia viable para ser incluida dentro del sistema de transporte público integrado de la ciudad de 
Bogotá, con el fin de dignificar el transporte y proporcionar a la población con discapacidad física un servicio de transporte accesible, de calidad y con amplia cobertura que les garantice el derecho de movilizarse libremente por el territorio distrital. Asimismo, se empleó como metodología el diseño de campo y descriptiva.

Las conclusiones del estudio demuestran que al garantizar la accesibilidad al transporte público urbano para todas las personas incluyendo a las que presentan alguna discapacidad, aumenta la calidad de vida de la población, porque el sistema de transporte público se convierte en el medio por el cual se accede a oportunidades sociales, económicas y culturales necesarias para consolidar un vínculo participativo dentro de la sociedad.

Se puede afirmar de lo anteriormente reseñado, en cuanto al transporte urbano y la movilidad urbana, muestra que es ineludible tener una visión integral de los problemas para llegar a las soluciones más adecuadas. Por lo que, sería interesante investigar con mucho más profundidad el proceso de la toma de decisiones sobre la movilidad de personas y el transporte urbano, puesto que tienen una serie de impactos sociales, tecnológicos, ambientales, económicos, financieros y culturales que juegan un papel importante sobre los ciudadanos, e influyen profundamente en la vida cotidiana y las relaciones de las personas.

\section{Metodología}

El estudio se encuentra sustentado en un método mixto, es decir una investigación exploratoria el cual permite brindar la aproximación de la realidad de la movilidad en bus como sistema de transporte urbano en Bogotá, partiendo de la base de que el tema en estudio ha sido poco tratado, es decir el acercamiento inicial, lo cual es necesario para ser estudiado de manera 
más precisa. Asimismo, el estudio es de enfoque explicativo, puesto que a través del tema de la movilidad urbana se logra descubrir la realidad del transporte público en Bogotá (Hernández, Fernández y Baptista, 2010).

En este sentido, se asume que la investigación se direcciona al abordaje de la movilidad urbana, lo cual construye, modifica, articula procesos experienciales que están en permanente cambio, por lo que el estudio se realiza en un contexto social determinado como lo es la ciudad de Bogotá. En definitiva, los procedimientos desarrollados para la realización del presente artículo científico son los siguientes:

- Se realiza un análisis de los antecedentes anteriores a esta investigación que pudieran estar relacionados con el tema, Trabajos Especiales de Grados, tesis.

- Se hace una revisión de la literatura existente acerca del tema.

- Se revisan documentos y registros existentes que coadyuvan a tener una visión más

clara.

- Se procede a la elaboración del presente marco metodológico tomando en cuenta los recursos disponibles existentes (Fulentes primarias, secundarias, bibliográficas, legales, documentos, trabajos anteriores y fuentes electrónicas - páginas Web como mera referencia).

- Seguidamente, se describen las conclusiones y las recomendaciones. 


\section{Marco teórico}

\section{Aspecto del Transporte Público}

A continuación, Rodríguez y Mojica (2008), exponen los aspectos que se derivan del transporte público:

Aspectos sociales: Contribuyen a una política de transporte, en donde los aspectos sociales cobran fuerza en cuanto la equidad, accesibilidad, salud y bienestar general de una población, y a su vez, puede tener efectos sobre la economía e incluso el medio ambiente de una ciudad.

Aspectos económicos: La eficiencia de una buena gestión urbana refleja el bienestar de toda la ciudadanía, pues si un ciudadano se desplaza solamente durante 30 minutos para llegar a su lugar de trabajo, su tiempo de desplazamiento (en la mayoría de las situaciones) es de una hora diaria, lo que se traduce en una situación adecuada, y cualquier duración menor a una hora es una ganancia.

Aspectos ambientales: Los grandes efectos del cambio climático siguen siendo uno de los problemas ambientales del presente siglo XXI, donde el transporte es un factor determinante en la mejoría o empeoramiento de la situación actual debido a las emisiones de dióxido de carbono $\left(\mathrm{CO}_{2}\right)$.

Transporte y movilidad urbana se refiere a todas las formas en las que los individuos se movilizan dentro de las ciudades, ya sea transporte público, privado, a pie, entre otras. En este sentido, un sistema de transporte público sostenible es aquel que moviliza a los ciudadanos; que no ponga en riesgo la calidad de vida de las personas que lo utilizan (Rodríguez y Targa, 2004).

AGLALA ISNN 2215-7360

2015; 6 (1): 102-120 
En las últimas décadas se ha observado un boom de las construcciones de infraestructuras viales, pensando que esto solucionaría las grandes concentraciones y embotellamiento del tráfico en Bogotá. Sin embargo, se ha comprobado que estas construcciones tienen consecuencias no deseadas, ya que al haber una nueva vía, se construyen centros comerciales y viviendas cerca de ella, la gente busca utilizarla más; lo cual conduce nuevamente al embotellamiento y al tráfico (Vega, 2004). El problema del transporte en suma, no se limita únicamente a mayores construcciones viales, sino es un tema mucho más complejo que involucra al Estado, pero también a la sociedad civil, puesto que implica un cambio de hábitos y prácticas por parte de los ciudadanos.

Teniendo claro que el transporte público es una de las opciones sostenibles a implementar en una ciudad, sólo puede funcionar efectivamente, sin convertirse en un instrumento de dominación y mantenimiento del statu quo (Hidalgo, 2009). Por tanto, es importante que pueda combinarse con políticas públicas de transporte público, posibilitando el desplazamiento de los ciudadanos. Adicionalmente, el transporte público es significativo debido a que a través del mismo se logra adecuar a una buena calidad de vida en la ciudad, asegurando además una movilidad acorde, bien sea con poder ir a pie, usar la bicicleta y con un buen sistema de transporte público colectivo (Robles, Ñañez y Quijano, 2009).

\section{Movilidad y modo de transporte}

Existe muchas características relevantes en cuanto a los principales factores que interfieren en la movilidad de las personas: el ingreso, el género, la edad, la ocupación y el nivel educacional. En primer lugar, la movilidad aumenta con el aumento del ingreso, seguidamente 
en segundo lugar, varía conforme a las características económicas y sociales de las personas. Por ejemplo, así como los hombres suelen viajar más que las mujeres, también los hombres adultos involucrados en actividades laborales se desplazan más que los habitantes jóvenes y de edad avanzada (Thomson y Bull, 2002).

A través de todo ello el nivel educacional de las personas se ve influenciado por el nivel de ingresos, por lo que el papel de peatones, ciclistas y usuarios de transporte público son las personas con bajos ingresos.

Por tanto, el Banco Mundial (2013) afirma que el ingreso tiene una influencia en la elección de los modos de desplazamiento, tal como se puede observar a modo de ejemplo en la figura 1 que la variación del uso de los modos de transporte en función del ingreso en la región metropolitana de São Paulo en 2007, observándose además que el uso del transporte no motorizado es más alto en las familias de bajos ingresos y disminuye cuando el ingreso se eleva. Por ello, el uso del transporte público parte desde un nivel bajo en las familias de escasos ingresos, familias de ingresos medianos, y las familias de ingresos altos (que pasan a usar el automóvil), por lo que el uso del transporte privado crece a medida que aumenta el ingreso.

Lo anteriormente descrito es un indicativo de que el uso de los modos de transporte e ingreso familiar afecta el estrato socioeconómico, puesto que las familias numerosas de bajos ingresos no pueden permitirse el uso del transporte público colectivo para todos sus miembros, debido a que gastaría buena parte o la totalidad del ingreso familia. De igual forma, un mal servicio de transporte público afecta a las familias pobres, puesto que dependen por completo del servicio público colectivo.

AGLALA ISNN 2215-7360

2015; 6 (1): 102-120 


\section{El Sistema Integrado de Transporte Público (SITP)}

La implantación del Sistema Integrado de Transporte Público (SITP), sigue siendo el proyecto más ambicioso de la ciudad en el corto y mediano plazo, implantándose con el servicio rápido y de buena calidad en toda la ciudad, siendo éste el reto más importante de la ciudad en materia de transporte público. En efecto, con este sistema se busca integrar el sistema de transporte masivo (TransMilenio) con el de transporte colectivo, por una parte que el primero sea la columna vertebral del sistema integrado y por el otro actúe como distribuidor y complemento del actual sistema de transporte en Bogotá (Perdomo, Mendoza, Mendieta y Baquero, 2010).

De igual forma, con el Sistema Integrado de Transporte Público será necesario eliminar las actuales empresas de transporte afiliadoras y reemplazarlas por empresas operadoras modernas y eficientes. Además, requiere la construcción de talleres en varias partes de la ciudad, de manera que se elimine el parqueo de los buses en las calles y se posibilite de verdad la administración del parque automotor.

Por ello, se debe eliminar todos los buses viejos; reestructurar las rutas, para dar un mejor servicio a toda la ciudad, y transformarlas en complementarias de TransMilenio. Asimismo, es indispensable integrar los sistemas tarifas integrales que darán lugar a contratos de duración definida entre el Distrito y las empresas operadoras, tomando en consideración las diferentes acciones de la administración distrital para bien de la toda ciudad, por lo que una evaluación de Transmilenio arroja una amplia gama de ventajas y resultados positivos después de algo más que dos años de experiencia:

AGLALA ISNN 2215-7360

2015; 6 (1): 102-120 
Los buses son amplios y cómodos, tienen un servicio hasta las 11 p.m. de la noche, las estaciones son agradables, poseen buena iluminación y son seguras. Adicionalmente, se puede afirmar que la construcción del sistema es mucho más económico que un metro o un tren ligero, puesto que se integrará las rutas y cubrirá toda la ciudad, desde los barrios y pueblos del área metropolitana.

Asimismo, con el sistema integrado de transporte público se ha logrado una integración tarifaria mediante un tiquete que permite la circulación de manera ilimitadamente. Otra de las ventajas de TransMilenio es el mejoramiento de las condiciones laborales para los conductores, puesto que esto ha permitido emplear también a mujeres como conductores mientras en el transporte público colectivo tradicional los conductores son exclusivamente hombres, cerca de la mitad de los mil empleos son mujeres, en todos los niveles y actividades de la empresa, un gran progreso si uno evalúa el sistema con criterios de equidad de género.

La importancia que supone el sistema de transporte público como elemento de inclusión social hace que las personas más vulnerables utilicen diferentes alternativas para tratar de reducir sus costes de movilidad, puesto que la práctica de todas estas opciones como un recurso para hacer frente a los altos costes en transporte que debe financiar la población en situación de pobreza pone de manifiesto la necesidad que este colectivo tiene que desplazarse a largas distancias para desarrollar sus actividades cotidianas, por lo que el sistema de transporte público tiene como fin último de inclusión la reducción de la situación de pobreza y exclusión social. Por tal razón, la adopción de estas alternativas facilita el uso del transporte público, permitiéndoles a las personas acceder a los diferentes servicios y oportunidades que ofrece la ciudad y su integración en las dinámicas de la ciudad (Behrentz, 2012).

AGLALA ISNN 2215-7360

2015; 6 (1): 102-120 
De igual manera, el sistema de transporte público posee una importancia en la sociedad donde los niveles de bienestar aumentando las posibilidades culturales y sociales de todas las personas. Además, mediante las relaciones sociales se hace posible el desarrollo de las infraestructuras separando los lugares de trabajo y residencia.

Por tal motivo, los desplazamientos que realizan las personas dentro de una ciudad tienen un coste, midiendo a su vez, las variables económicas y no económicas que logran afectar al bienestar de la colectividad. Adicionalmente, el coste generalizado en cuanto a infraestructura de transporte se convierte en una principal variable influyendo en las decisiones de los individuos en cuanto a los modos de elección del transporte público.

En todo caso, una mejora en la infraestructura del transporte público permitirá una disminución del coste generalizado del viaje, específicamente por el ahorro de tiempo que supone la tarifa y sus mejoras. Aunado a ello, repercute en una mejora del transporte público, suponiendo que el ahorro de tiempo de viaje representa un punto de beneficio en cuanto a la valoración de la rentabilidad en lo que se refiere a la inversión de la infraestructura.

Lo importante de esta materia es que las mejoras en la infraestructura de transporte hacen referencia a la congestión vehicular, que puede afectar a la mayor parte de población. Puesto que, la incorporación de vehículos en la vía de circulación incrementan los costes asociados al tiempo de desplazamiento, además la solución al problema de la congestión se debe centrar en la ampliación de la capacidad en la infraestructura vial.

En el ámbito económico, la infraestructura supo0ne una parte de gran relevancia en lo que ha movilidad y transporte público, debido a que facilita la cohesión social, admitiendo que una adecuada dotación de infraestructura vial aumenta la eficiencia del sistema productivo, 
estimula la inversión privada y mejora la competitividad de la economía constituyendo un sólido desarrollo económico a largo plazo.

\section{Conclusiones}

Al analizar la movilidad de las personas en las ciudades se enfatiza que ésta se realiza en diferentes circunstancias de comodidad, seguridad y tiempo, por lo que el uso excesivo del transporte privado hace necesario el fomento del transporte público colectivo. Se trata pues, que los retos actuales de organización del transporte urbano pasan por determinar el lugar que debe ocupar el automóvil y la accesibilidad tarifaria, temporal y territorial del transporte público colectivo, por lo que se trata de una cuestión de cohesión social y territorial del espacio urbano. Se puede constatar, que si el sistema de transporte tiene una gran afinidad puede llegar a conseguir que un área metropolitana tenga identidad territorial propia. Por tanto, el problema de la organización del transporte urbano en las grandes ciudades, no debe ser la atribución de funciones y responsabilidades que está delimitada en regulación de Bogotá, sino la política que se pretenda impulsar y los objetivos que se consigan alcanzar.

El transporte público presenta condiciones diversificadas de utilización si se compara con otros medios colectivos de consumo, debido a que éste puede ser apropiado tanto en forma colectiva como individual, quedando claro que el sistema de transporte público involucra una socialización de infraestructura (vías) y de medios de transporte (vehículos), mientras que el transporte individual involucra la misma socialización de la infraestructura, pero con la privatización del modo de transporte (bicicleta o automóvil). Aunado a ello, se puede constatar que otra característica de la estructura de circulación como la escasez de espacio que debe ser

AGLALA ISNN 2215-7360

2015; 6 (1): 102-120 
compartido entre los usuarios, hace al consumo real colectivo y sujeto a reglas colectivas, puesto que las velocidades, dimensiones y desempeños operacionales diversos entre personas y vehículos llevan a formas y tasas muy distintas de consumo de espacio y tiempo, que requieren un análisis de equidad.

El mantenimiento financiado por los ingresos municipales generales sería la base sistemática y segura para el mantenimiento de vías como alta prioridad en la estrategia de transporte urbano dentro de la ciudad de Bogotá. Por consiguiente, a medida que las ciudades se expanden las ciudades necesitan una cantidad básica de espacio para la circulación adecuado a su tamaño para operar eficientemente, bajo una planificación temprana y una infraestructura de transporte idóneo como requerimiento estratégico.

Estas serían las razones fundamentales por las que debe reconocerse que la dinámica de la expansión de la ciudad logra sus objetivos cuando posee una adecuada asignación y buena planificación del espacio destinado al movimiento. Por ello, la construcción de infraestructura vías debe ajustarse a la capacidad vial para sostener la expansión de la ciudad con políticas claras y estrictas sobre el uso y asignación del espacio vial para ajustar la expansión de la ciudad con la calidad de vida.

A partir de lo expuesto anteriormente, el estudio resulta un aporte de gran relevancia para el ámbito de la Ingeniería civil, puesto que pretende explicar los cambios en materia de movilidad urbana; lo cual promoverá el desarrollo de los ciudadanos e incremento del bienestar en su vida cotidiana. Por tal razón, el estudio representa un alcance y una oportunidad para reflexionar sobre el modelo urbano, poniendo en interrelación diferentes políticas sectoriales de movilidad y buscando la cohesión entre las diferentes políticas ya emprendidas por la gestión

AGLALA ISNN 2215-7360

2015; 6 (1): 102-120 
municipal de Bogotá. Por último, es conveniente anotar que las normativas son los fundamentos para que la movilidad urbana se haga una realidad, y los planes, programas y estrategias establecidos por la municipalidad de Bogotá se logren obtener un sistema de transporte público, eficiente, equitativo, accesible para todos y de calidad.

\section{Referencias bibliográficas}

Ávila, A. (2003). Transporte urbano e inclusâo social: elementos para políticas públicas. Brasília, IPEA. Instituro de Pesquisa Econômica Aplicada.

Banco Mundial. (2013). Transforming Cities with Transit: Transit and Land. Use Integration for Sustainable Urban Development, EUA. 2013.

Banco Mundial (2009). World Development Report, 2009: Reshaping Economic Geography Washington, D.C.

Figueroa, I. y Castañeda, J. (2013). Caracterización de los criterios logísticos clave de transporte urbano de pasajeros para el Barrio Belisario Caicedo de la Comuna 20 en Santiago de Cali. Universidad Autónoma de Occidente.

Hernández, R., Fernández, C. y Baptista, P. (2010). Metodología de la investigación (Quinta edición ed.). México D.F.: Mc Graw Hill.

Hidalgo, D. y Yepes, T. (2004). Are bus rapid transit systems effective in poverty reduction? Experience of Bogota's Transmilenio and lessons for other cities. Transportation Research $84^{\text {th }}$ Annual Board Meeting.

Martínez, D. (2012). Estrategias para promover la accesibilidad, cobertura y calidad en el sistema de transporte público urbano para la población con discapacidad física: Caso Bogotá. Universidad Nacional de Colombia.

AGLALA ISNN 2215-7360

2015; 6 (1): 102-120 
Miralles-Guasch, C y Cebollada, A. Movilidad y transporte: opciones políticas para la ciudad Barcelona. Recuperado el 4 de enero de 2013 de http://mobilitat.uab.es/Arxius_comuns/Publicacions/2003-

Alternativas_MovyTr_gemott.pdf (citado el).

Perdomo, J. A., Castañeda, H., y Mendieta, J. C. (2010). Evaluación de impacto de las Fases I y II del sistema de transporte masivo TransMilenio sobre el tiempo total de desplazamiento de los usuarios del transporte público tradicional en Bogotá. Documentos CEDE 2010-11.

Perdomo, J. A., Mendoza, C., Mendieta, J. C. y Baquero, A. F. (2007). Study of the Effect of the TransMilenio Mass Transit Project on the Value of Properties in Bogotá. Colombia. Lincoln Institute of Land Policy, Working Paper.

Ribeiro, et al. (2000). Transporte e mudanças climáticas, Mauad Editora e Coppe/UFRJ, Río de Janeiro.

Robles, D., Ñañez, P. y Quijano, N. (2009). Control y simulación de tráfico urbano en Colombia: Estado del arte. Revista de Ingeniería, Vol. 2 (29).59-69.

Rodríguez, D. y Mojica, C. Land Value Impacts of Bus Rapid Transit. The case of Bogotá's TransMilenio. Land Lines, Li (2008). ncoln Institute of Land Policy.

Rodríguez, D. A. y Targa, F. (2004). Value of Accessibility to Bogotá's Bus Rapid Transit System. Transport Reviews, Vol. 24 (5).587-610.

Rogat, Jorge (2009). Planificación e implementación de un sistema de bus rápido en América Latina: resumen orientado a tomadores de decisiones. Bogotá, Colombia: Investigaciones de Espacio.

AGLALA ISNN 2215-7360

2015; 6 (1): 102-120 
Thomson I. y Bull A. (2002). La congestión del tránsito urbano: causas y consecuencias económicas y sociales" en Revista de la cepal No 76 P. 109 A 121.

Vega, Pablo (2004). Movilidad (espacial) y vida cotidiana en contextos de metropolización. En Debates en Sociología, número 28. Lima. 2004. 